The Free Internet Journal for Organic Chemistry
Paper

Arkivoc 2019, part v, 133-141

Organic Chemistry

\title{
A simple route for synthesis of 5-(furan-3-yl)barbiturate/thiobarbiturate derivatives via a multi-component reaction between arylglyoxals, acetylacetone and barbituric/thiobarbituric acid
}

Fatemeh Dehghanzadeh, Fereshteh Shahrokhabadi, and Mohammad Anary-Abbasinejad*

Department of Chemistry, Faculty of Science, Vali-e-Asr University of Rafsanjan, Rafsanjan 77176, Iran

E-mail: m.anary@vru.ac.ir

Received 12-05-2018

Accepted 02-10-2019

Published on line 03-03-2019

\section{Abstract}

An effective protocol for the synthesis of 5-(furan-3-yl)barbiturate and 5-(furan-3-yl)thiobarbiturate derivatives through a one-pot three-component reaction of readily available starting materials arylglyoxals, barbituric acid or thiobarbituric acid and acetylacetone in water as solvent is reported.

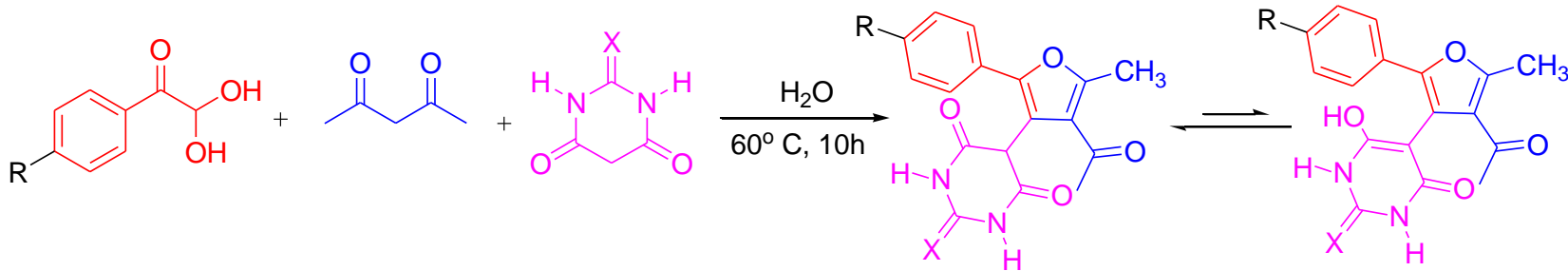

Keywords: Multi-component reactions, arylglyoxal, acetylacetone, barbituric acid, thiobarbituric acid, furan 


\section{Introduction}

Barbituric and 2-thiobarbituric acids are well-known classes of organic compounds with a wide variety of pharmacological activities which have found applications as the main skeleton for a series of barbiturate / thiobarbiturate drugs used as antioxidants, hypnotics, anticonvulsants, sedatives, anaesthetics, antifungal, and central nervous system depressants. ${ }^{1-3}$ Combination of barbituric/thiobarbituric acid moieties with other pharmacophoric moieties may result in new types of scaffold with potential biological activities. Many efforts have been made in fusing barbiturates and thiobarbiturates with other molecular skeletons such as 1,3diketones, ${ }^{4,5}$ isatins, $^{6-8}$ Meldrum's acid, ${ }^{9}$ 4-hydroxycoumarin ${ }^{10}$ and pyrroles. ${ }^{11}$ Barbiturates have also been widely used in the manufacturing of plastics, ${ }^{12}$ textiles, $^{13}$ and polymers. ${ }^{14}$

Furan moieties are important substructures that have been found in numerous natural products, such as kailolides ${ }^{15}$ and combranolides. ${ }^{16}$ These heterocycles are also found in a variety of commercial products such as pharmaceuticals, fragrances, and dyes. ${ }^{17}$

Multi-component reactions (MCRs), especially those conducted in water, offer significant advantages over conventional linear-type syntheses, because the reaction components combine with each other in a single step to generate new products. On the other hand, the low cost, and the lack of inflammability, explosive, and carcinogenic properties of water are some of the economic and environmental benefits of using water as solvent. $^{18,19}$

Arylglyoxals with a carbonyl group adjacent to the aldehyde functionality, are reactive and versatile species which have been widely used for the synthesis of various heterocyclic and carbocyclic compounds. Arylglyoxals have been recently reported as the key component in several multi-component reactions for connecting reaction components to each other to make the main skeleton of the product. ${ }^{20-22}$

We have recently focused our attention on developing new multi-component reactions of arylglyoxals for the synthesis of new heterocyclic compounds. ${ }^{23-25}$ In continuation of these works, here we report a new threecomponent reaction of arylglyoxals with acetylacetone and barbituric or thiobarbituric acid for the synthesis of a series of new polyfunctionalized 5-(furan-3-yl)barbiturates and 5-(furan-3-yl)thiobarbiturates.

\section{Results and Discussion}

In order to investigate the three-component reaction of arylglyoxals, acetylacetone and barbituric acid, at first we studied the reaction between 4-methoxyphenylglyoxal monohydrate 1a, acetylacetone 2 and barbituric acid 3a in water as solvent (Scheme 1). A mixture of 4-methoxyphenylglyoxal monohydrate 1a and acetylacetone 2 was stirred in water at $60^{\circ} \mathrm{C}$. After thirty minutes barbituric acid was added and the mixture was heated at $60^{\circ} \mathrm{C}$ for 10 hours more. TLC analysis of the reaction mixture showed the presence of only one product. Silica-gel chromatography afforded 5-[4-acetyl-2-(4-methoxyphenyl)-5-methylfuran-3-yl]pyrimidine$2,4,6(1 H, 3 H, 5 H)$-trione $4 a$ in $90 \%$ yield.

To investigate the scope of the reaction, different arylglyoxals were treated with acetylacetone and barbituric acid and the corresponding 5-furyl-3-barbiturates 4a-g were obtained in good yields (Table 1). Next, reactions were carried out between acetylacetone, arylglyoxals and thiobarbituric acid in water at similar conditions and the corresponding 5-(furan-3-yl)thiobarbiturates $4 \mathrm{~h}-\mathrm{m}$ were obtained in good yields. 
<smiles></smiles>

Scheme 1. Synthesis of 5-[4-acetyl-2-(4-methoxyphenyl)-5-methylfuran-3-yl]pyrimidine-2,4,6(1H,3H,5H)-trione $4 a$ by three-component reaction between 4-methoxyphenylglyoxal monohydrate, acetylacetone and barbituric acid.

Table 1. Three-component reaction between arylglyoxals, barbituric acid or thiobarbituric acid and acetylacetone for synthesis of 5-(furan-3-yl)barbiturate/thiobarbiturate derivatives<smiles></smiles>

\begin{tabular}{|c|c|c|c|c|c|}
\hline $4,4^{\prime}$ & $\mathrm{R}$ & $x$ & Product & $\mathrm{mp}{ }^{\circ} \mathrm{C}$ & Yield \% \\
\hline a & OMe & 0 & & $300-303$ & 90 \\
\hline b & $\mathrm{H}$ & $\mathrm{O}$ & & $309-311$ & 78 \\
\hline c & $\mathrm{Me}$ & 0 & & $274-276$ & 86 \\
\hline d & $\mathrm{Cl}$ & 0 & & $298-300$ & 90 \\
\hline e & $\mathrm{C}_{4} \mathrm{H}_{4}$ & 0 & & $279-281$ & 94 \\
\hline
\end{tabular}


Table 1. Continued

\begin{tabular}{|c|c|c|c|c|c|}
\hline $4,4^{\prime}$ & $\mathrm{R}$ & $x$ & Product & $\mathrm{mp}{ }^{\circ} \mathrm{C}$ & Yield \% \\
\hline$f$ & $\mathrm{Br}$ & 0 & & 297-299 & 88 \\
\hline g & $\mathrm{NO}_{2}$ & 0 & & $280-282$ & 90 \\
\hline h & OMe & $\mathrm{S}$ & & $275-277$ & 90 \\
\hline $\mathbf{i}$ & $\mathrm{H}$ & $\mathrm{S}$ & & $279-281$ & 95 \\
\hline j & $\mathrm{Br}$ & $\mathrm{S}$ & & $264-266$ & 78 \\
\hline k & $\mathrm{NO}_{2}$ & $\mathrm{~S}$ & & $229-231$ & 80 \\
\hline I & Me & $\mathrm{S}$ & & $273-275$ & 90 \\
\hline m & $\mathrm{Cl}$ & $S$ & & $261-263$ & 85 \\
\hline
\end{tabular}

The structures of compounds $4 a-m$ were inferred from their elemental analyses and their IR, ${ }^{1} H$ NMR, and ${ }^{13} \mathrm{C}$ NMR spectroscopic data. Compounds $4 a-m$ may exist as two tautomers 4 or 4 ' (Table 1). The tautomerism of barbituric acid and thiobarbituric acid derivatives has been extensively studied. These studies showed that 
5-substituted barbiturates usually exist as the keto form in polar solvents such as DMSO in contrast to 5substituted thiobarbiturates which exist mainly as the enol form in polar solvents. ${ }^{26,27}$ The NMR spectra of compounds $\mathbf{4 a - 4 f}$ showed that those compounds which include the barbiturate moiety in their structures existed mainly as keto tautomer 4 in DMSO- $d_{6}$ solution. In contrast, compounds $\mathbf{4 h}-\mathbf{4 m}$ with a thiobarbiturate moiety in their structure existed as the enol tautomer 4'. The NMR spectra of compound $\mathbf{4 g}$ with nitrophenyl and barbiturate moieties showed the presence of two isomers in nearly equal amounts. The $500-\mathrm{MHz}{ }^{1} \mathrm{H} \mathrm{NMR}$ spectrum of $4 a$ exhibited three sharp signals at $\delta 2.19,2.47,3.72$, ppm for two methyl groups and one methoxy. The $\mathrm{CH}$ proton of the barbiturate moiety resonated as a singlet signal at $4.93 \mathrm{ppm}$. This signal was not observed at the ${ }^{1} \mathrm{H}$ NMR spectrum of thiobarbiturate derivative $\mathbf{4 h}$, which showed that this compound exists as the enol form. The aromatic protons of 4 a resonated as two doublet signals at 6.88 and $7.54 \mathrm{ppm}$. Two NH protons were observed at $9.22 \mathrm{ppm}$ as a broad signal. The ${ }^{13} \mathrm{C} \mathrm{NMR}$ spectrum of compound 4 a showed fifteen distinct signals in agreement with the proposed structure. The $\mathrm{CH}$ carbon of the barbiturate moiety resonated at $40.89 \mathrm{ppm}$. In derivatives that are in the enol form, such as $\mathbf{4 h}$, this carbon was observed at about $90 \mathrm{ppm}$. The structural assignments made on the basis of the NMR spectra of compound 4a were supported by its IR spectrum. The amide carbonyl groups exhibited a strong absorption band at about 1666 $\mathrm{cm}^{-1}$. The ketone carbonyl was observed at $1701 \mathrm{~cm}^{-1}$ as a strong absorption band.

The suggested mechanism for formation of furanyl barbiturate/thiobarbiturate derivatives $4 a-m$ by the reaction between arylglyoxals, acetylacetone and barbituric/thiobarbituric acids is shown in Scheme 2 . The Michael addition of barbituric/thiobarbituric acid to the Intermediate obtained from Knoevenagel condensation of arylglyoxals with acetylacetone afforded reactive 1,4-diketone 6 . The Paal-Knorr cyclization of this intermediate afforded product 4.

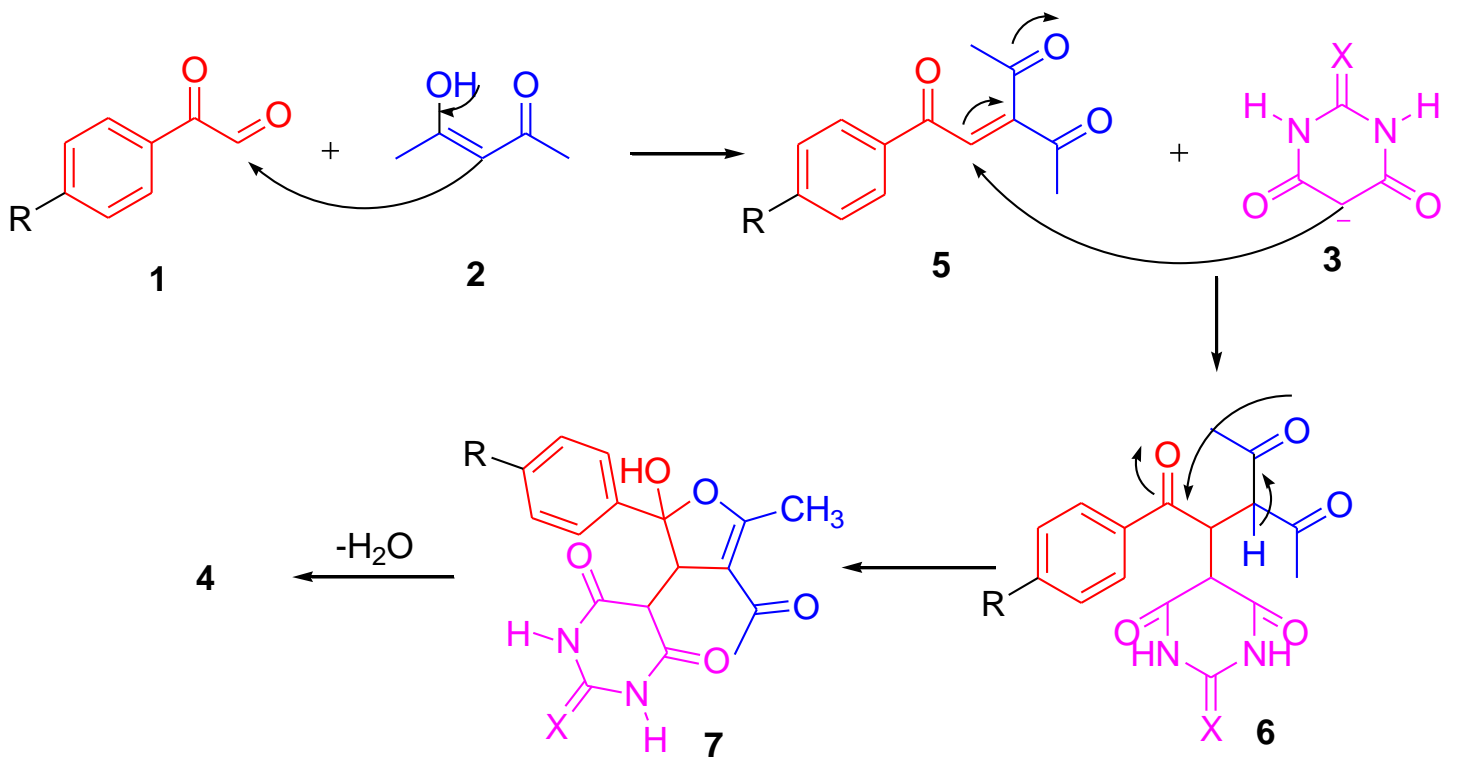

Scheme 2. Suggested mechanism for formation of 5-(furan-3-yl)barbiturate/thiobarbiturate derivatives 4a-m

\section{Conclusions}

In conclusion, we report a simple three-component reaction between arylglyoxal monohydrates, acetylacetone and barbituric or thiobarbituric acid for the synthesis of polyfunctionalized 5-(furan-3- 
yl)barbiturates and 5-(furan-3-yl)thiobarbiturate derivatives in good yields. The method employs readily available starting materials, neutral reaction conditions and water as an environmentally green solvent.

\section{Experimental Section}

General. All solvents and chemicals except arylglyoxals were purchased from commercial sources and used without further purification. The utilized arylglyoxals were prepared by the $\mathrm{SeO}_{2}$-oxidation of the related aryl methylketones on the basis of the reported procedure, and used as their monohydrates. ${ }^{28}$ Melting points were determined on a Melt-Tem II melting point apparatus and are uncorrected. IR spectra were recorded on a Shimadzu IR-470 spectrometer. All of the NMR spectra were recorded on a Varian model UNITY Inova 500 $\mathrm{MHz}\left({ }^{1} \mathrm{H}\right.$ : $\left.500{ }^{13} \mathrm{C}: 125 \mathrm{MHz}\right) \mathrm{NMR}$ spectrometer. Chemical shifts of ${ }^{1} \mathrm{H},{ }^{13} \mathrm{C} N M R$ are reported in parts per million (ppm) from tetramethylsilane (TMS) as an internal standard in DMSO- $d_{6}$ as a solvent.

General procedure. A mixture of arylglyoxal $(1 \mathrm{mmol})$ and acetylacetone $(1 \mathrm{mmol})$ in water $(15 \mathrm{~mL})$ was stirred at $60{ }^{\circ} \mathrm{C}$ for $20 \mathrm{~min}$. Then, barbituric or thiobarbituric acid $(1 \mathrm{mmol})$ was added to this mixture. The reaction mixture was then stirred at $60{ }^{\circ} \mathrm{C}$ for $10 \mathrm{~h}$ more. The solvent was removed under reduced pressure, and the residue was purified by column chromatography on silica-gel, using EtOAc-EtOH mixture (7:1) as eluent.

5-[4-Acetyl-2-(4-methoxyphenyl)-5-methylfuran-3-yl]pyrimidine-2,4,6(1H,3H,5H)-trione (4a). Dark yellow powder, mp 300-303 ${ }^{\circ} \mathrm{C}$ (Yield: 90\%). IR (KBr) v $v_{\max } / \mathrm{cm}^{-1}: 1666,1701(\mathrm{C}=0), 3424(\mathrm{NH}) .{ }^{1} \mathrm{H}-\mathrm{NMR}(500 \mathrm{MHz}$ DMSO- $\left.d_{6}\right): \delta$ (ppm): $2.19\left(3 \mathrm{H}, \mathrm{s}, \mathrm{CH}_{3}\right), 2.47\left(3 \mathrm{H}, \mathrm{s}, \mathrm{CH}_{3}\right), 3.72\left(3 \mathrm{H}, \mathrm{s}, \mathrm{OCH}_{3}\right), 4.93(\mathrm{H}, \mathrm{s}, \mathrm{CH}), 6.88\left(2 \mathrm{H}, \mathrm{d}^{3} \mathrm{~J}_{\mathrm{HH}} 8.8\right.$ $\mathrm{Hz}, 2 \mathrm{CH}, \mathrm{Ar}), 7.55\left(2 \mathrm{H}, \mathrm{d},{ }^{3} \mathrm{~J}_{\mathrm{HH}} 8.8 \mathrm{~Hz}, 2 \mathrm{CH}, \mathrm{Ar}\right), 9.22(2 \mathrm{H}, \mathrm{s}, 2 \mathrm{NH}) .{ }^{13} \mathrm{C}-\mathrm{NMR}\left(125 \mathrm{MHz} \mathrm{DMSO}-d_{6}\right): \delta(\mathrm{ppm}): 14.7$, 28.9, 40.9, 55.5, 114.0, 115.8, 125.0, 126.0, 126.7, 147.9, 152.7, 154.8, 158.3, 164.3, 196.5. Calcd. for $\mathrm{C}_{18} \mathrm{H}_{16} \mathrm{~N}_{2} \mathrm{O}_{6}: \mathrm{C}, 60.67 ; \mathrm{H}, 4.53 ; \mathrm{N}, 7.86 \%$. Found: $\mathrm{C}, 60.55 ; \mathrm{H}, 4.67 ; \mathrm{N}, 7.93 \%$.

5-[4-Acetyl-5-methyl-2-phenylfuran-3-yl]pyrimidine-2,4,6(1H,3H,5H)-trione (4b). Light orange powder, $\mathrm{mp}$ 309-311 ${ }^{\circ} \mathrm{C}$ (Yield: 78\%). IR ( $\left.\mathrm{KBr}\right) v_{\max } / \mathrm{cm}^{-1}: 1654(\mathrm{C}=\mathrm{O}), 1694(\mathrm{C}=\mathrm{O}), 3199(\mathrm{NH}) .{ }^{1} \mathrm{H}-\mathrm{NMR}\left(500 \mathrm{MHz} \mathrm{DMSO}-d_{6}\right): \delta$ (ppm): $2.41\left(3 \mathrm{H}, \mathrm{s}, \mathrm{CH}_{3}\right), 2.72\left(3 \mathrm{H}, \mathrm{s}, \mathrm{CH}_{3}\right), 5.12(\mathrm{H}, \mathrm{s}, \mathrm{CH}), 7.43-7.56(5 \mathrm{H}, \mathrm{m}, \mathrm{HAr}), 11.21(2 \mathrm{H}, \mathrm{s}, 2 \mathrm{NH}) .{ }^{13} \mathrm{C}-\mathrm{NMR}$ (125 MHz DMSO- $\left.d_{6}\right): \delta$ (ppm): 15.8, 30.3, 47.5, 113.4, 121.4, 125.1, 127.3, 129.0, 129.5, 151.7, 152.4, 159.1, 169.1, 194.8. Calcd. for $\mathrm{C}_{34} \mathrm{H}_{28} \mathrm{~N}_{4} \mathrm{O}_{10}: \mathrm{C}, 62.57 ; \mathrm{H}, 4.32 ; \mathrm{N}, 8.59 \%$. Found: $\mathrm{C}, 62.55 ; \mathrm{H}, 4.37 ; \mathrm{N}, 8.53 \%$.

5-[4-Acetyl-5-methyl-2-(p-tolyl)furan-3-yl]pyrimidine-2,4,6(1H,3H,5H) (4c). Light pink powder, mp $274-276{ }^{\circ} \mathrm{C}$ (Yield: 86\%). IR (KBr) v $v_{\max } / \mathrm{cm}^{-1}: 1645,1718(\mathrm{C}=\mathrm{O}), 3218(\mathrm{NH}) .{ }^{1} \mathrm{H}-\mathrm{NMR}(500 \mathrm{MHz}$ DMSO-d 6 ): $\delta(\mathrm{ppm}): 2.34(3 \mathrm{H}$, $\left.\mathrm{s}, \mathrm{CH}_{3}\right), 2.40\left(3 \mathrm{H}, \mathrm{s}, \mathrm{CH}_{3}\right), 2.71\left(3 \mathrm{H}, \mathrm{s}, \mathrm{CH}_{3}\right), 5.07(\mathrm{H}, \mathrm{s}, \mathrm{CH}), 7.29\left(2 \mathrm{H}, \mathrm{d}^{3}{ }^{3} \mathrm{~J}_{\mathrm{HH}} 8.2 \mathrm{~Hz}, 2 \mathrm{CH}, \mathrm{HAr}\right), 7.44\left(2 \mathrm{H}, \mathrm{d},{ }^{3} \mathrm{~J}_{\mathrm{H}} 8.2\right.$ $\mathrm{Hz}, 2 \mathrm{CH}, \mathrm{HAr}), 11.21(2 \mathrm{H}, \mathrm{s}, 2 \mathrm{NH}) .{ }^{13} \mathrm{C}-\mathrm{NMR}\left(125 \mathrm{MHz}\right.$ DMSO-d $\left.d_{6}\right): \delta$ (ppm): 15.8, 21.3, 30.3, 47.5, 112.8, 121.4, 127.2, 129.7, 130.0, 139.0, 151.7, 152.5, 158.8, 169.1, 194.8. Calcd. for $\left(\mathrm{C}_{18} \mathrm{H}_{16} \mathrm{~N}_{2} \mathrm{O}_{5}\right): \mathrm{C}, 63.52 ; \mathrm{H}, 4.74 ; \mathrm{N}$, 8.23\%. Found: C, 63.45; $\mathrm{H}, 4.87 ; \mathrm{N}, 8.27 \%$.

5-[4-Acetyl-2-(4-chlorophenyl)-5-methylfuran-3-yl]pyrimidine-2,4,6(1H,3H,5H)-trione (4d). Light yellow powder, mp 298-300 ${ }^{\circ} \mathrm{C}$ (Yield: 87\%). IR (KBr) $v_{\text {max }} / \mathrm{cm}^{-1}:$ 1650, $1708(\mathrm{C}=\mathrm{O}), 3202(\mathrm{NH}) .{ }^{1} \mathrm{H}-\mathrm{NMR}(500 \mathrm{MHz}$ DMSO- $\left.d_{6}\right): \delta$ (ppm): $2.41\left(3 \mathrm{H}, \mathrm{s}, \mathrm{CH}_{3}\right), 2.72\left(3 \mathrm{H}, \mathrm{s}, \mathrm{CH}_{3}\right), 5.13(\mathrm{H}, \mathrm{s}, \mathrm{CH}), 7.48\left(2 \mathrm{H}, \mathrm{d}^{3}{ }^{3} \mathrm{JHH}_{\mathrm{HH}} 8.5 \mathrm{~Hz}, 2 \mathrm{CH}, \mathrm{HAr}\right), 7.52$ $\left(2 \mathrm{H}, \mathrm{d},{ }^{3} \mathrm{~J}_{\mathrm{HH}} 8.5 \mathrm{~Hz}, 2 \mathrm{CH}, \mathrm{HAr}\right), 11.26(2 \mathrm{H}, \mathrm{s}, 2 \mathrm{NH}) .{ }^{13} \mathrm{C}-\mathrm{NMR}(125 \mathrm{MHz}$ DMSO-d $)$ : $\delta$ (ppm): 15.8, 30.3, 47.5, 114.0, 126.7, 127.8, 129.0, 129.5, 134.1, 151.2, 151.7, 159.4, 168.9, 194.8. Calcd. for $\left(\mathrm{C}_{17} \mathrm{H}_{13} \mathrm{ClN}_{2} \mathrm{O}_{5}\right): \mathrm{C}, 56.60$; H, 3.63; N, 7.77\%. Found: C, 56.45; H, 3.75; N, 7.56\%.

5-[4-Acetyl-5-methyl-2-(naphthalen-2-yl)furan-3-yl]pyrimidine-2,4,6(1H,3H,5H)-trione (4e). Light brown powder, mp $279-281{ }^{\circ} \mathrm{C}$ Yield: 94\%. IR (KBr) v max $/ \mathrm{cm}^{-1}$ : 1643, 1715 (C=O), $3622(\mathrm{NH}) .{ }^{1} \mathrm{H}-\mathrm{NMR}$ (500 MHz DMSO$\left.d_{6}\right): \delta(p p m): 2.43\left(3 \mathrm{H}, \mathrm{s}, \mathrm{CH}_{3}\right), 2.76\left(3 \mathrm{H}, \mathrm{s}, \mathrm{CH}_{3}\right), 5.31(\mathrm{H}, \mathrm{s}, \mathrm{CH}), 7.50-7.52(\mathrm{H}, \mathrm{m}, \mathrm{HAr}), 7.55-7.57(\mathrm{H}, \mathrm{m}, \mathrm{HAr})$, 
7.69-7.71 (H, m, HAr), 7.55-7.86 (2H, d, ${ }^{3} \mathrm{~J}_{\mathrm{HH}}$ 7.5, HAr), 7.95-7.97 (H, m, HAr), 8.02 (H, d, $\left.{ }^{3} \mathrm{~J}_{H H} 8.5, \mathrm{HAr}\right), 8.09(\mathrm{H}$, s, HAr), $11.26(2 \mathrm{H}, \mathrm{s}, 2 \mathrm{NH}) .{ }^{13} \mathrm{C}-\mathrm{NMR}\left(125 \mathrm{MHz}\right.$ DMSO- $\left.d_{6}\right): \delta$ (ppm): 15.9, 30.319, 47.7, 113.9, 121.6, 124.8, $126.2,126.5,127.2,127.3,128.1,128.8,129.1,133.1,133.2,151.8,152.3,159.4,169.1$, 194.8. Calcd. for $\left(\mathrm{C}_{21} \mathrm{H}_{16} \mathrm{ClN}_{2} \mathrm{O}_{5}\right): \mathrm{C}, 67.02 ; \mathrm{H}, 4.28 ; \mathrm{N}, 7.44 \%$. Found: $\mathrm{C}, 67.25 ; \mathrm{H}, 3.95 ; \mathrm{N}, 7.56 \%$.

5-[4-Acetyl-2-(4-bromophenyl)-5-methylfuran-3-yl]pyrimidine-2,4,6(1H,3H,5H)-trione (4f). Light pink powder, mp 297-299 ${ }^{\circ} \mathrm{C}$ (Yield: 92\%). IR (KBr) v $\max / \mathrm{cm}^{-1}: 1706,1768(\mathrm{C}=0), 3273(\mathrm{NH}) .{ }^{1} \mathrm{H}-\mathrm{NMR}(500 \mathrm{MHz}$ DMSO- $\left.d_{6}\right): \delta(p p m): 2.40\left(3 \mathrm{H}, \mathrm{s}, \mathrm{CH}_{3}\right), 2.71\left(3 \mathrm{H}, \mathrm{s}, \mathrm{CH}_{3}\right), 5.12(\mathrm{H}, \mathrm{s}, \mathrm{CH}), 7.49\left(\mathrm{H}^{3}{ }^{3} \mathrm{~J}_{\mathrm{HH}} 8.5 \mathrm{~Hz}, \mathrm{HAr}\right), 7.69\left(\mathrm{H},{ }^{3} \mathrm{~J}_{\mathrm{HH}}\right.$ $8.5 \mathrm{~Hz}, \mathrm{HAr}), 11.25(2 \mathrm{H}, \mathrm{s}, 2 \mathrm{NH}) .{ }^{13} \mathrm{C}-\mathrm{NMR}\left(125 \mathrm{MHz}\right.$ DMSO-d $\left.d_{6}\right): \delta(\mathrm{ppm}): 15.8,30.3,47.5,114.1,121.5,122.7$, 129.2, 132.2, 132.5, 151.3, 151.7, 159.4, 168.9, 194.8. Calcd. for $\left(\mathrm{C}_{17} \mathrm{H}_{13} \mathrm{BrN}_{2} \mathrm{O}_{5}\right): \mathrm{C}, 50.39 ; \mathrm{H}, 3.23 ; \mathrm{N}, 6.91 \%$. Found: $\mathrm{C}, 50.45 ; \mathrm{H}, 3.32 ; \mathrm{N}, 6.95 \%$

5-[4-Acetyl-5-methyl-2-(4-nitrophenyl)furan-3-yl]pyrimidine-2,4,6(1H,3H,5H)-trione and 5-[4-acetyl-5methyl-2-(4-nitrophenyl)furan-3-yl]-6-hydroxypyrimidine-2,4(1H,3H)-dione $(\mathbf{4 g})$. Orange powder, $\mathrm{mp} 280$ $282{ }^{\circ} \mathrm{C}$ (Yield: 90\%). IR (KBr) v $v_{\text {max }} / \mathrm{cm}^{-1}$ : 1663, 1697 (C=O), $3233(\mathrm{NH}), 3481(\mathrm{OH}) .{ }^{1} \mathrm{H}-\mathrm{NMR}\left(500 \mathrm{MHz}\right.$ DMSO- $\left.d_{6}\right)$ : $\delta$ (ppm): $2.23\left(3 \mathrm{H}, \mathrm{s}, \mathrm{CH}_{3}\right), 2.43\left(3 \mathrm{H}, \mathrm{s}, \mathrm{CH}_{3}\right), 2.62\left(3 \mathrm{H}, \mathrm{s}, \mathrm{CH}_{3}\right), 2.76\left(3 \mathrm{H}, \mathrm{s}, \mathrm{CH}_{3}\right), 5.28(\mathrm{H}, \mathrm{s}, \mathrm{CH}), 7.27\left(\mathrm{H},{ }^{3} \mathrm{~J}_{\mathrm{HH}} 9.0\right.$ $\mathrm{Hz}, \mathrm{HAr}), 7.82\left(\mathrm{H}^{3}{ }^{3} \mathrm{HH}_{\mathrm{HH}} 8.9 \mathrm{~Hz}, \mathrm{HAr}\right), 8.27\left(\mathrm{H}^{3}{ }^{3} \mathrm{H}_{\mathrm{HH}} 9.0 \mathrm{~Hz}, \mathrm{HAr}\right), 8.32\left(\mathrm{H}^{3}{ }^{3} \mathrm{~J}_{\mathrm{HH}} 8.9 \mathrm{~Hz}, \mathrm{HAr}\right), 10.90(\mathrm{H}, \mathrm{s}, \mathrm{NH}), 11.30$ $(2 \mathrm{H}, \mathrm{s}, 2 \mathrm{NH}) .{ }^{13} \mathrm{C}-\mathrm{NMR}\left(125 \mathrm{MHz}\right.$ DMSO- $\left.d_{6}\right): \delta$ (ppm): 15.1, 15.8, 29.8, 29.3, 47.5, 83.4, 115.3, 116.6, 121.8, 124.7, 125.5, 125.8, 127.9, 134.9, 136.7, 146.3, 147.0, 147.5, 150.1, 150.7, , 151.6, 158.8, 160.6, 168.6, 194.5, 194.8. Calcd. for $\left(\mathrm{C}_{17} \mathrm{H}_{13} \mathrm{~N}_{3} \mathrm{O}_{7}\right)$ : C, 54.99; $\mathrm{H}, 3.53 ; \mathrm{N}, 11.32 \%$. Found: $\mathrm{C}, 54.95 ; \mathrm{H}, 3.52 ; \mathrm{N}, 11.49 \%$.

\section{5-[4-Acetyl-2-(4-methoxyphenyl)-5-methylfuran-3-yl]-6-hydroxy-2-thioxo-2,3-dihydropyrimidin-4(1H)-one}

(4h). Brown powder, mp 275-277 ${ }^{\circ} \mathrm{C}$ (Yield: 90\%). IR (KBr) $v_{\max } / \mathrm{cm}^{-1}: 1703,1741(\mathrm{C}=\mathrm{O}), 3423(\mathrm{OH}, \mathrm{NH}) .{ }^{1} \mathrm{H}-$ NMR (500 MHz DMSO-d $)$ : $\delta(p p m): 2.22\left(3 \mathrm{H}, \mathrm{s}, \mathrm{CH}_{3}\right), 2.59\left(3 \mathrm{H}, \mathrm{s}, \mathrm{CH}_{3}\right), 3.74\left(3 \mathrm{H}, \mathrm{s}, \mathrm{OCH}_{3}\right), 6.98\left(\mathrm{H}, \mathrm{d}^{3} \mathrm{~J}_{\mathrm{HH}} 8.8\right.$ $\mathrm{Hz}, \mathrm{HAr}), 7.41\left(\mathrm{H}, \mathrm{d},{ }^{3} \mathrm{~J}_{\mathrm{HH}} 8.8 \mathrm{~Hz}, \mathrm{HAr}\right), 12.28(1 \mathrm{H}, \mathrm{bs}, \mathrm{NH}), 12.31(1 \mathrm{H}, \mathrm{s}, \mathrm{NH}) .{ }^{13} \mathrm{C}-\mathrm{NMR}\left(125 \mathrm{MHz} \mathrm{DMSO}-d_{6}\right): \delta$ (ppm): 15.1, 30.0, 55.6, 89.4, 108.4, 114.7, 123.1, 124.8, 126.8, 128.8, 149.4, 156.8, 159.4, 160.6, 174.4, 194.3. Calcd. for $\left(\mathrm{C}_{18} \mathrm{H}_{16} \mathrm{~N}_{2} \mathrm{O}_{5} \mathrm{~S}\right)$ : C, 58.05; $\mathrm{H}, 4.33 ; \mathrm{N}, 7.52 ; \mathrm{S}, 8.61 \%$. Found: $\mathrm{C}, 58.19 ; \mathrm{H}, 4.14 ; \mathrm{N}, 7.56 ; \mathrm{S}, 8.71 \%$.

5-(4-Acetyl-5-methyl-2-phenylfuran-3-yl)-6-hydroxy-2-thioxo-2,3-dihydropyrimidin-4(1H)-one (4i). Light pink powder, mp 279-281 ${ }^{\circ} \mathrm{C}$ (Yield: 95\%). IR (KBr) $\mathrm{v}_{\max } / \mathrm{cm}^{-1}:$ 1709, $1744(\mathrm{C}=\mathrm{O}), 3138(\mathrm{OH}, \mathrm{NH}) .{ }^{1} \mathrm{H}-\mathrm{NMR}(500 \mathrm{MHz}$ DMSO- $\left.d_{6}\right): \delta(p p m): 2.23\left(3 \mathrm{H}, \mathrm{s}, \mathrm{CH}_{3}\right), 2.61\left(3 \mathrm{H}, \mathrm{s}, \mathrm{CH}_{3}\right), 7.30\left(\mathrm{H}, \mathrm{d}^{3}{ }^{3} \mathrm{~J}_{\mathrm{HH}} 7.7 \mathrm{~Hz}, \mathrm{HAr}\right), 7.40\left(\mathrm{H}, \mathrm{t},{ }^{3} \mathrm{~J}_{\mathrm{HH}} 7.7 \mathrm{~Hz}, \mathrm{HAr}\right)$, $7.49\left(2 \mathrm{H}, \mathrm{d},{ }^{3} \mathrm{~J}_{\mathrm{HH}} 7.7 \mathrm{~Hz}, 2 \mathrm{CH}, \mathrm{HAr}\right), 12.29(2 \mathrm{H}, \mathrm{s}, 2 \mathrm{NH}) .{ }^{13} \mathrm{C}-\mathrm{NMR}\left(125 \mathrm{MHz}\right.$ DMSO-d $\left.d_{6}\right): \delta(p p m): 15.1,30.0,89.1$, 110.3, 124.9, 125.2, 128.3, 129.2,129.4, 130.5, 149.1, 157.3, 160.6, 174.4, 194.3. Calcd. for $\left(\mathrm{C}_{17} \mathrm{H}_{14} \mathrm{~N}_{2} \mathrm{O}_{4} \mathrm{~S}\right): \mathrm{C}$, 59.64; H, 4.12; N, 8.18; S, 9.37\%. Found: C, 59.45; H, 4.27; N, 8.03; S, 9.44\%.

5-[4-Acetyl-2-(4-bromophenyl)-5-methylfuran-3-yl]-6-hydroxy-2-thioxo-2,3-dihydropyrimidin-4(1H)-one (4j). Orange powder, mp 264-266 ${ }^{\circ} \mathrm{C}$ (Yield: 78\%). IR (KBr) v $\max / \mathrm{cm}^{-1}: 1645(\mathrm{C}=\mathrm{O}), 3201(\mathrm{OH}, \mathrm{NH}) .{ }^{1} \mathrm{H}-\mathrm{NMR}(500 \mathrm{MHz}$ DMSO- $\left.d_{6}\right): \delta(p p m): 2.25\left(3 \mathrm{H}, \mathrm{s}, \mathrm{CH}_{3}\right), 2.60\left(3 \mathrm{H}, \mathrm{s}, \mathrm{CH}_{3}\right), 5.16(1 \mathrm{H}, \mathrm{bs}, \mathrm{OH}), 7.44\left(2 \mathrm{H}, \mathrm{d}^{3}{ }^{3} \mathrm{JHH}_{\mathrm{H}} 8.1 \mathrm{~Hz}, \mathrm{HAr}\right), 7.60$ $\left(2 \mathrm{H}, \mathrm{d},{ }^{3} \mathrm{~J}_{\mathrm{HH}} 8.1 \mathrm{~Hz}, \mathrm{HAr}\right), 11.68(\mathrm{H}, \mathrm{s}, \mathrm{NH}), 12.35(\mathrm{H}, \mathrm{s}, \mathrm{NH}) .{ }^{13} \mathrm{C}-\mathrm{NMR}(125 \mathrm{MHz}$ DMSO-d $)$ : $\delta$ (ppm): 15.1, 30.0, 88.9, 111.1, 121.4, 125.0, 127.1, 129.7, 132.2, 132.4, 148.1, 157.7, 160.7, 174.4, 194.3. Calcd. for $\left(\mathrm{C}_{17} \mathrm{H}_{13} \mathrm{BrN}_{2} \mathrm{O}_{4} \mathrm{~S}\right): \mathrm{C}, 48.47 ; \mathrm{H}, 3.11 ; \mathrm{N}, 6.65 ; \mathrm{S}, 7.61 \%$. Found: $\mathrm{C}, 48.25 ; \mathrm{H}, 3.17 ; \mathrm{N}, 6.73 ; \mathrm{S}, 7.54 \%$.

5-[4-Acetyl-5-methyl-2-(4-nitrophenyl)furan-3-yl]-6-hydroxy-2-thioxo-2,3-dihydropyrimidin-4(1H)-one (4k). Yellow powder, mp 229-231 ${ }^{\circ} \mathrm{C}$ (Yield: 80\%). IR (KBr) $v_{\max } / \mathrm{cm}^{-1}: 1695,1744(\mathrm{C}=0), 3473(\mathrm{OH}, \mathrm{NH}) .{ }^{1} \mathrm{H}-\mathrm{NMR}(500$ MHz DMSO-d $\left.d_{6}\right): \delta(p p m): 2.25\left(3 \mathrm{H}, \mathrm{s}, \mathrm{CH}_{3}\right), 2.63\left(3 \mathrm{H}, \mathrm{s}, \mathrm{CH}_{3}\right), 5.01(1 \mathrm{H}, \mathrm{bs}, \mathrm{OH}), 7.76\left(2 \mathrm{H}, \mathrm{d}^{3}{ }^{3} \mathrm{HH}_{\mathrm{H}} 8.8 \mathrm{~Hz}, \mathrm{HAr}\right)$, $8.27\left(2 \mathrm{H}, \mathrm{d},{ }^{3} \mathrm{~J}_{\mathrm{HH}} 8.8, \mathrm{HAr}\right), 12.16(2 \mathrm{H}, \mathrm{s}, 2 \mathrm{NH}) .{ }^{13} \mathrm{C}-\mathrm{NMR}\left(125 \mathrm{MHz}\right.$ DMSO- $\left.d_{6}\right): \delta(\mathrm{ppm}): 15.1,30.0,88.0,112.7$, $120.9,124.7,125.6,127.4,136.6,146.3,146.8,158.7,160.6,174.5,194.4$. Calcd. for $\left(\mathrm{C}_{17} \mathrm{H}_{13} \mathrm{~N}_{3} \mathrm{O}_{6} \mathrm{~S}\right): \mathrm{C}, 52.71$; H, 3.38; N, 10.85; S, 8.28\%. Found: C, 52.45; H, 3.34; N, 10.83; S, 8.10\%.

5-[4-Acetyl-5-methyl-2-(p-tolyl)furan-3-yl]-6-hydroxy-2-thioxo-2,3-dihydropyrimidin-4(1H)-one (4I). Light orange powder, mp 273-275 ${ }^{\circ} \mathrm{C}$ (Yield: 90\%). IR (KBr) $v_{\max } / \mathrm{cm}^{-1}: 1706,1742$ (C=O), $3411(\mathrm{OH}, \mathrm{NH}) .{ }^{1} \mathrm{H}-\mathrm{NMR}$ 
(500 MHz DMSO-d $\left.d_{6}\right): \delta(p p m): 2.23\left(3 \mathrm{H}, \mathrm{s}, \mathrm{CH}_{3}\right), 2.28\left(3 \mathrm{H}, \mathrm{s}, \mathrm{CH}_{3}\right), 2.60\left(3 \mathrm{H}, \mathrm{s}, \mathrm{CH}_{3}\right), 7.21\left(2 \mathrm{H}, \mathrm{d},{ }^{3} \mathrm{~J}_{\mathrm{HH}} 8.0 \mathrm{~Hz}\right.$, HAr), 7.37(2H, d, $\left.{ }^{3} \mathrm{~J}_{\mathrm{HH}} 8.0 \mathrm{~Hz}, \mathrm{HAr}\right), 12.26(2 \mathrm{H}, \mathrm{s}, 2 \mathrm{NH}) .{ }^{13} \mathrm{C}-\mathrm{NMR}\left(125 \mathrm{MHz}\right.$ DMSO- $\left.d_{6}\right): \delta$ (ppm): 15.1, 21.3, 30.0, 89.2, 109.5, 124.9, 125.2, 127.1, 127.8, 129.7, 137.8, 149.4, 157.1, 160.6, 174.4, 194.3. Calcd.for $\left(\mathrm{C}_{18} \mathrm{H}_{16} \mathrm{~N}_{2} \mathrm{O}_{4} \mathrm{~S}\right)$ : C, 60.66; H, 4.53; N, 7.86; S, 9.00\%. Found: C, 60.44; H, 4.54; N, 7.93; S, 8.79\%.

\section{5-[4-Acetyl-2-(4-chlorophenyl)-5-methylfuran-3-yl]-6-hydroxy-2-thioxo-2,3-dihydropyrimidin-4(1H)-one}

(4m). Light pink powder, mp 261-263 ${ }^{\circ} \mathrm{C}$ (Yield: 85\%). IR ( $\left.\mathrm{KBr}\right) \mathrm{v}_{\max } / \mathrm{cm}^{-1}: 1713,1748(\mathrm{C}=\mathrm{O}), 3144(\mathrm{OH}, \mathrm{NH}) .{ }^{1} \mathrm{H}-$ NMR (500 MHz DMSO- $\left.d_{6}\right): \delta(p p m): 2.23\left(3 \mathrm{H}, \mathrm{s}, \mathrm{CH}_{3}\right), 2.61\left(3 \mathrm{H}, \mathrm{s}, \mathrm{CH}_{3}\right), 7.47-7.51(4 \mathrm{H}, \mathrm{m}, \mathrm{HAr}), 12.30(\mathrm{H}, \mathrm{s}, \mathrm{NH})$, $12.34(\mathrm{H}, \mathrm{s}, \mathrm{NH}) .{ }^{13} \mathrm{C}-\mathrm{NMR}\left(125 \mathrm{MHz}\right.$ DMSO- $\left.d_{6}\right): \delta(\mathrm{ppm}): 15.1,30.0,88.76,111.1,125.0,126.8,129.31,129.3$, 129.5, 132.8, 148.0, 157.6, 160.6, 174.4, 194.2. Calcd.for $\left(\mathrm{C}_{17} \mathrm{H}_{13} \mathrm{ClN}_{2} \mathrm{O}_{4} \mathrm{~S}\right): \mathrm{C}, 54.19 ; \mathrm{H}, 3.48 ; \mathrm{N}, 7.43 ; \mathrm{S}, 8.51$. Found: C, 54.16; H, 3.44; N, 7.47; S, 8.55\%.

\section{Acknowledgements}

We gratefully acknowledge financial support from the Vail-e-Asr University of Rafsanjan Faculty Research Grant.

\section{Supplementary Material}

The ${ }^{1} \mathrm{H}$ NMR and ${ }^{13} \mathrm{C}$ NMR data for compounds $4 a-m$ associated with this article can be found in the online version.

\section{References}

1. Rathee, P.; Tonk, R. K.; Dalal, A.; Ruhil, M. K.; Kumar, A. Org. Cell. Mol. Biol. 2016, 62, 5.

2. Mobinikhaledi, A.; Kalhor, M. Int. J. Drug Dev. Res. 2010, 2, 268.

3. Mohamed, N. R.; El-Saidi, M. M. T.; Ali, Y. M.; Elnagdi, M. H. Bioorg. Med. Chem. 2007, 15, 6227. https://doi.org/10.1016/i.bmc.2007.06.023

4. Li, J.; Shi, W.; Yang, W.; Kang, Z.; Zhang, M.; Song, L. RSC Adv. 2014, 4, 29549. https://doi.org/10.1039/C4RA03199G

5. Khalafi-Nezhad, A.; Panahi, F. Synthesis 2011, 6, 984. https://doi.org/10.1055/s-0030-1258446

6. Soleimani, E.; Ghorbani, S.; Ghasempour, H. R. Tetrahedron 2013, 69, 8511. https://doi.org/10.1016/j.tet.2013.06.080

7. Safaei, H. R.; Shekouhy, M.; Rahmanpur, S.; Shirinfeshan, A. Green Chem. 2012, 14, 1696. https://doi.org/10.1039/c2gc35135h

8. Deng, J.; Mo, L. P.; Zhao, F. Y.; Zhang, Z. H. ACS Comb. Sci. 2012, 14, 335. https://doi.org/10.1021/co3000264

9. Azzam, S. H. S.; Pasha, M. A. Tetrahedron Lett. 2012, 53, 7056. https://doi.org/10.1016/j.tetlet.2012.10.056

10. Kazemi-Rad, R.; Azizian, J.; Kefayati, H. Tetrahedron Lett. 2014, 55, 6887. https://doi.org/10.1016/i.tetlet.2014.10.099 
11. Dommaraju, Y.; Prajapati, D. Mol. Divers. 2015, 19, 173. https://doi.org/10.1007/s11030-014-9547-1

12. Bartzatt, R. J. Pharm. Biomed. Anal. 2002, 29, 909. https://doi.org/10.1016/S0731-7085(02)00168-1

13. McClenaghan, N. D.; Absalon, C.; Bassani, D. M. J. Am. Chem. Soc. 2003, 125, 13004. https://doi.org/10.1021/ja0372098

14. Look, S. A.; Burch, M. T.; Fenical, W.; Qi-tai, Z.; Clardy, J. J. Org. Chem. 1985, 50, 5741. https://doi.org/10.1021/jo00350a061

15. Fenical, W.; Okeeda, R. K.; Basnadurraga, M. M.; Culver, P.; Jacobs, R. S. Science 1981, 212, 1512. https://doi.org/10.1126/science.6112796

16. Hou, X. L.; Cheung, H. Y.; Hon, T. U.; Kwan, P. L.; Lo, T. H.; Tong, S. Y.; Wong, H. N. C. Tetrahedron 1998, 54, 1955 and references cited therein. https://doi.org/10.1016/S0040-4020(97)10303-9

17. Candeias, N. R.; Cal, P. M.; André, V.; Duarte, M. T.;Veiros, L. F.; Gois, P. M.Tetrahedron 2010, 66, 2736. https://doi.org/10.1016/j.tet.2010.01.084

18. Pirrung, M. C.; Sarma. K. D. J. Am. Chem. Soc. 2004, 126, 444. https://doi.org/10.1021/ja038583a

19. Jiang, B.; Li, Q. Y.; Zhang, H.; Tu, S. J.; Pindi, S.; Li, G. Org, Lett. 2012, 14, 700. https://doi.org/10.1021/ol203166c

20. Jiang, B.; Yi, M. S.; Shi, F.; Tu, S. J.; Pindi, S.; Mc Dowell, P.; Li, G. Chem. Commun. 2012, $48,808$. https://doi.org/10.1039/C1CC15913E

21. Eftekhari-Sis, B.; Zirak, M.; Akbari, A. Chem. Rev. 2013, 113, 2958. https://doi.org/10.1021/cr300176g

22. Anary-Abbasinejad, M ; Talebizadeh, M. J. Iran. Chem. Soc. 2014, 11, 963. https://doi.org/10.1007/s13738-013-0362-x

23. Mousavizadeh, F.; Talebizadeh, M.; Anary-Abbasinejad, M. Tetrahedron Lett. 2018, 59, 2970. https://doi.org/10.1016/j.tetlet.2018.06.043

24. Masoudi, M.; Anary-Abbasinejad, M. TetrahedronLett. 2016, 57, 103. https://doi.org/10.1016/j.tetlet.2015.11.075

25. Oguz, S. F.; Dogan I. Spect. Lett. 2004, 37, 607. https://doi.org/10.1081/SL-200037606

26. Bojarski, J. T.; Mokrosz. J. L.; Barton, H. J.; Paluchowska, M. H. Adv. Heterocycl. Chem. 1985, $38,229$. https://doi.org/10.1016/S0065-2725(08)60921-6

27. Riley, H. A.; Gray, A. R. Org. Synth. 1943, 2, 509. 